\title{
Repetitive strain injuries in key-board operators
}

The work of Key-board operators was initially associated to Repetitive Strain Injuries (RSI) in Japan around 1960, when these workers complained of pain in the neck-shoulderarm-hand are called Occupational Cervicobrachial Disorders by the committe of the Japan Associations of Industrial Health. In Australia, an increase of RSI in keyboard operators was registered around 1970. In Brazil, cases of tenosynovitis, a clinical type of RSI, related to key-board's work since 1982, have been registered.

In 1986, the Labour Union of the Data Processing Companies for the State of São Paulo requested from the Labour Ministry of São Paulo the development of an Inspection Program for these companies. In the planning of how these inspections would be run, it was decided to use the CAT (Work Accidents Comunication) of tenosynovitis registered at Social Welfare.

As the National Institute of Medical Assistance and Social Welfare established in November 1986 tenosynovitis as an occupational disease, the period select for the CATs analysis was from November 1986 to December 1987. This study analysed 284 CATs registered in the city of São Paulo. The name RSI was not used by the Social Welfare Department in 1987.

The information analysed regarded the business, the worker (age, sex, marital status, occupation, wages) and the accident characterization (medical service and diagnosis). The data was processed by the Computer Service of Preventive Medicine, Department of the São Paulo University Medical School.

Summarizing, the information on the workers gathered was: $79,9 \%$ were women; the majority $(60,8 \%)$ were 25 to 34 years of age; $43,9 \%$ were unmarried and $48,9 \%$ were married; $71,9 \%$ received between 4 to 6 minimun salaries.
We compared the information collected through the CATs with the 1989 Annual Social Information Report (RAIS) of the Labour Ministry. In that year, there were 12.272 keyboard operators working. In relation to sex, we observed that $52,7 \%$ were women. It seems to be that the diseasis more common in women. In relation to the age, according to RAIS's data $35,7 \%$ of the keyboard operators were between 18 to 24 years of age and those with tenosynovitis represented only $13,5 \%$ suggesting that the more serious cases of the disease occured 5 years after working in this occupation.

The occupation collected through the CATs in $91,8 \%$ were of keyboard operators. This information differs from international literature, that report more RSI in assembly-line operators. The explanation of this fact is the mobilization of data processing workers in Brazil to recognize RSI as an occupational disease.

The keyboard operation can be associated with RSI because of the following factors: high speed finger work totaling 12.000 strokes per hour associated with production prizes; continuous working periods with a break time after three hours; frequent over-time; poor ergonomic design of the furniture and machines causing muscular strain; increase of stress because of electronic monitoring of production; environments with low temperature and inadequate lighting; use of force to stroke and 12 hour work periods.

The recognition of tenosynovitis as an occupational disease was mainly done by Work Accident Experts of the Social Welfare Department that were distributed in 5 areas of the city. Most patients were attended at the Central Agency $(70,6 \%)$ because of its localization. Medical Services of the Companies were responsible for attending $11,8 \%$.

The main diagnosis was for the right arm $(90,1 \%)$; $59,4 \%$ for the upper members; tenosynovitis of the wrist 
occured in $21,8 \%$ of the cases. This observation consolidates that the best name is RSI which explains the generalized attack of the superior members.

In the relation to the duration of leaves, only $6,7 \%$ tookless than 15 days off. The majority took off more than 90 days, making it evident that only the more serious cases had been registered.

The keyboard operators with tenosynovitis in general directed themselves to orthopedists of medical health plans (common in data processing business) who recommended they stay away from work from 7 to 10 days. After the treatment, the keyboard operators returned to work without alterations in the production. These episodes many times went unkown by to Medical Services of the Companies because the medical certificates were filed in the Personnel Department. In these cases, they did not file CATs because the business is responsible for the payment of the first 15 days away from the job.

The CATs data and the inspection at the companies contributed to changing the Ergonomic Guidelines of the Labour Ministry, establishing prevention measures for the disease through the Portaria 3751 of November 23, 1990. 